\title{
Index Vol. 20, 1989
}

\begin{tabular}{|c|c|c|} 
No. & $\mathbf{1}$ & $\mathbf{2}$ \\
$\begin{array}{c}\text { Month } \\
\text { Issue }\end{array}$ & $\begin{array}{c}\text { January } \\
\text { Physics }\end{array}$ & $\begin{array}{c}\text { February } \\
\text { Physics }\end{array}$ \\
Pages & $1-16$ & $17-32$
\end{tabular}

Applied Physics, Physics in Industry ACAPPI, Officers, 105

Nice meeting, 60

Europhysics Industrial Workshops

EIW-4, ion beams, 32

EIW-5, structural ceramics, 150

EIW-6, conducting polymers, 150

Astronomy and Astrophysics

Division and Section Boards, 106

Solar newsletter, 135

European astrophysics network, 51-52

Globular star clusters, dynamics, 163-166

Hipparcos not a write-off, 134

IRAM array operational, 155

Prize, poster, 112

Student, 22

Supernova 1987 A, 13-16

Tethered satellites, 19-22

Atomic and Molecular Physics

Division and Section Boards, 106

Presentation to Chairman, 113

Electron scattering with photoelectrons, 64-66

lon-neutral collisions at low energies, 124-126

Size-selected neutral clusters, properties of, 41-44

Condensed Matter Physics

Annual conference, 60

Disordered materials, molecular dynamics

simulations, $69-72$

Division and Section Boards, 106-107

EMRS cooperation, 135

Heavy fermions and superconductivity, 159-162

ISIS, resource for neutron studies, 117, 119-123

Liquid physics meeting, 112

Nanocrystalline materials and nano-glasses, 130-133

Polymers, physical mechanisms in failure, 52-54

Electrically conducting, 139-142

Small clusters, physics of, 151-154

Superconductivity, high $\mathrm{T}_{\mathrm{c}}$ workshop, 31

Who's Who, 135

Conference listings, 33-38, 72, 144-149

Education

Computers in teaching, 68

Counsellors for China, 21

Employment, from academic physics to industry, 30-31

\section{Authors}

Aguilar-Benitez M., 92-95

Andreoni W., 151-154

Averbuch P., 30-31

Baeriswyl D., 31

Bartel W., 75

Bassi D., 124-126

Bickerton R., 1-5

\section{Nobel Prize 1989}

The 1989 Nobel Prize in Physics has been awarded to three physicists for work starting in the 1950's that led to recent advances in the field of atomic precision spectroscopy. The award winners are Norman Ramsey (Harvard, USA) for the application of separated oscillatory electromagnetic fields, Wolfgang Paul (University of Bonn, FRG) and Hans Dehmelt (University of Washington, USA) for the introduction and development of the ion trap technique. An appreciation will be published in the next issue of Europhysics News.

\begin{tabular}{c|c}
3 & 4 \\
March & April \\
Peetings & Physics \\
$33-40$ & $41-56$
\end{tabular}

\begin{tabular}{c|}
5 \\
May \\
Physics \\
$57-72$
\end{tabular}

6
June
LEP
$73-96$

\begin{tabular}{|c|c|c|c|}
\hline $7 / 8$ & 9 & 10 & $11 / 12$ \\
\hline July/Aug. & September & October & Nov./Dec. \\
\hline $\begin{array}{l}\text { Physics } \\
\text { +Directory }\end{array}$ & Physics & $\begin{array}{c}\text { Physics } \\
+ \text { Meetings }\end{array}$ & Physics \\
\hline $97-116$ & $117-136$ & $137-156$ & $157-176$ \\
\hline
\end{tabular}

\section{Principal Subjects}

European Physical Society

Action Committees, officers, 105

Associate Members, delegates, 103

Council, decisions, 56

Future meetings, 59

Meeting report, 57-59

Division and Section Boards, 106-107

Interdivisional Groups, 59, 107-108

Executive Committee, 56, 103

Decisions, 109

Financial situation, 18

General Conference, 145

IOM delegates, 32, 103

Meetings, sponsorship and approval, 143

Members, 103-105

New, 68, 129, 142

President's report, 17

IV Seminar on International Research Facilities, 59-60, 112

Southern European School, 138

Finland, to join CERN, 154

Fusion

Electro-chemical induced, 45, 63

Inertial confinement, 9

JET, latest results, 1-5

Muon-catalysed, 61-63

Superconducting magnets, 6-9

Hewlett-Packard Europhysics Prize

Call for nominations, 67

1989 Winners, 5, 159-162

Value increased, 162

High Energy and Particle Physics

Divisional Board, 107

EPS Prize, 112

ICFA plots new course, 54-55

LEP, $73,75-96,135,176$

Divisional comment, 75

Experimental programme, 75-79

Higgs connexion, 92-95

Machine design, 80-91

Quarks, lattices and transputers, 24-28

Second lepton family, 10-12

SSC go-ahead, 110

Japan, Phys. Soc., EPS privileges, 126

Froelich P., 61-63 Gleiter H., 130-133

Harbeke G., 135

Haubenreich P.N., 6-9

Heggie D.C., 163-166

Herrero J., 92-95

Heyvaerts J., 51

Jackson D., 68

Jaeglé P., 127-129
Karow H.U., 9 Kausch H.H., 52-54 Kempkens H., 46-50 Kienle P., 99-102 Lingeman E.W.A., 18 Lock W.O., 54-55 Mackintosh A.R., 67 Maeder A., 13-16 Mariani F., 19-22
Interdivisional Groups, Boards, 107-108

Accelerators, formation, 59

Computational Physics, elections, 5

Experimental Physics Control Systems, 110

History of Physics, formation, 59

Physics for Development, 111-112

Lasers, X-ray, 127-129

Photo-acoustic spectroscopy,

Nobel Prize in Physics

1988, L. Lederman, M. Schwartz, J. Steinberger, 10-12 1989, winners, 175

Nuclear Physics

Amsterdam pulse stretcher, 154

Divisional Board, 107

Strong field atomic processes, 97, 99-102

Optics, Divisional Board, 107

Plasma Physics (see also Fusion)

Divisional Board, 107

Hollow cathode arcs, 46-50

Publications

Action Committee, officers, 105

Editorial Boards, 108

EPS Recognized Journals, 108

Europhysics Conference Abstracts, 108

Streamlining publication procedures, 171-174

Subscription rates for 1990 ,

Quantum Electronics (see also Lasers)

Divisional Board, 107

Conference report, EQEC-2, 170

Schools, listings, $39-40,149-150$

Synchrotron radiation, ESRF Convention, 28-29

Third World, help, 111-112, 134

USSR Physical Society, 166

Yugoslav physics directory, 56

Monnier R., 31

Parrinello M., 69-72

Picasso E., 80-91

Ricci R.A., 17

Rodot M., 135

Salaneck W.R., 139-142

Schmidt W., 9

Sens J.C., 171-174

Shaw E.N., 45, 158
Sigrist M.W., 167-170 Steglich F., 159-162 Tosi P., 124-126 Turlay R., 75-79 Uhlenbusch J. 46-50

Winter K., 10-11

Woerdman J.P., 170 van Herwijnen E., 171-174

\section{Optronic Techniques in Diagnostic and Therapeutic Medicine \\ Florence, 26-27 March 1990 \\ Topics at the Conference will include: \\ - Light source technology - Optics of tissues \\ - Light-tissue interaction - Diagnostic techniques \\ - Therapeutic techniques. \\ Information from: \\ Dr. A. Somigli, Florence Optronics/1 \\ c/o Istituto Nazionale di Ottica, Largo E. Formi 6 \\ I-50100 Firenze \\ Conference Chairman: R. Pratesi, IEQ-CNR \\ Fax ++39 (55) 414612}

